\title{
Animated versus Static Views of Steady Flow Patterns
}

\author{
Colin Ware ${ }^{1}$ Daniel Bolan ${ }^{1}$ Ricky Miller ${ }^{1}$ David H. Rogers ${ }^{2} \quad$ James P. Ahrens $^{2}$ \\ ${ }^{1}$ University of New Hampshire $\quad{ }^{2}$ Los Alamos National Laboratory
}

\begin{abstract}
Two experiments were conducted to test the hypothesis that animated representations of vector fields are more effective than common static representations even for steady flow. We compared four flow visualization methods: animated streamlets, animated orthogonal line segments (where short lines were elongated orthogonal to the flow direction but animated in the direction of flow), static equally spaced streamlines, and static arrow grids. The first experiment involved a pattern detection task in which the participant searched for an anomalous flow pattern in a field of similar patterns. The results showed that both the animation methods produced more accurate and faster responses. The second experiment involved mentally tracing an advection path from a central dot in the flow field and marking where the path would cross the boundary of a surrounding circle. For this task the animated streamlets resulted in better performance than the other methods, but the animated orthogonal particles resulted in the worst performance. We conclude with recommendations for the representation of steady flow patterns.
\end{abstract}

Keywords: Flow visualization, animated flow, vector field visualization.

Concepts: • Human-centered computing $\sim$ Empirical studies in visualization;

\section{Introduction}

The visualization of $2 \mathrm{D}$ vector fields has applications including surface ocean currents, surface wind patterns as well as slices through electromagnetic fields. Significant effort has gone into determining the most effective method for statically representing these patterns, but the use of animation to show flows and other vector fields has not been previously evaluated despite the fact that this is also a common practice. There would seem to be no obvious reason to use animation to represent 2D steady flow patterns since there is no change over time; animation, is generally more costly to generate in terms of computation and more difficult to deliver to the viewer. Nevertheless, the fact that flow patterns inherently involve movement suggests that animation should be an intuitive mode of representation for such data. Also, it is possible that an animated version of a pattern may be easier to perceive than a statically represented version of the same pattern. Another possible advantage of animated streamlets is that they will perceptually

1'e-mail:cware@ccom.unh.edu

(c) 2016 Association for Computing Machinery. ACM acknowledges that this contribution was authored or co-authored by an employee, contractor or affiliate of the United States government. As such, the United States

Government retains a nonexclusive, royalty-free right to publish or reproduce this article, or to allow others to do so, for Government purposes only.

SAP '16, July 22-23, 2016, Anaheim, CA, USA

(C) 2016 ACM. ISBN 978-1-4503-4383-1/16/07 ..\$15.00

DOI: http://dx.doi.org/10.1145/2931002.2931012 interfere less with other background information compared to static visualization methods. The basis of this argument is that moving pattern processing and static pattern processing occurs in separate visual "channels" in the primary visual cortex of the human brain [Newsome and Pare, 1988; Livingstone and Hubel, 1987]. This paper reports on an empirical comparison of static and animated representation of simulated flow patterns.

\subsection{Effective display of vector fields}

Methods developed to represent two dimensional vector fields, include arrow grids, line integral convolution (LIC) and streamlines. Arrow grids have been used extensively for decades and are still the most common method used in practice despite studies suggesting that they are ineffective. Laidlaw et al. [2005] compared six different vector field display methods for a number of different tasks. For example, one task involved advection pathway tracing and for this they found that a method using fat headed arrows arranged along equally spaced streamlines was the most effective. Liu et al [2012] also found streamlines to be far better than arrows for a pattern recognition task; oriented line integral convolution also worked well. Ware [2008] proposed a perceptual theory of flow visualization, based on the idea that an effective flow visualization should selectively excite visual cortex neurons sensitive to patterns tangential to the direction of flow. Based on the theory he suggested that streamline based representations should be most effective for showing flow orientation. Pineo and Ware [2008] developed a computation model of the human visual system to evaluate this theory and the results broadly agreed with the empirical findings. In other work, a comparison of streamlines to a grid of wind barbs (glyphs used in meteorology) showed the streamline representation to be superior in enabling people to see flow patterns [Pilar and Ware 2013].

All of the evaluations we have discovered are of static representations suitable for print media. Yet many authors have proposed that animation should provide an effective method for flow visualization. Animation techniques include color table animation [Jobard and Lefer, 1997; Lefer et al. 2004; Laramee et al. 2004] where cycling through a color look up table causes a visual pattern to move over a field. The line integral convolution (LIC) method has also frequently been animated (e.g. [Berger and Groller, 2000; Telea and van Wijk, 1999]. Bachthaler and Weiskopf [2008] constructed an argument based on human perception that the spatial frequencies of the patterns animated using LIC are lower than optimal in the direction of flow. In order to remedy this they developed textures that were elongated orthogonally to the flow direction but which were animated in the direction of flow. In this way they were able to control the spatial and temporal frequency of the moving patterns. Other animation methods include the animation of conventional arrows, textures [Max and Becker 1996] and the animation of traces or points along streamlines or pathlines [Laramee, 2002; Sobel et al, 2004; Huber and Healey, 2005]. 

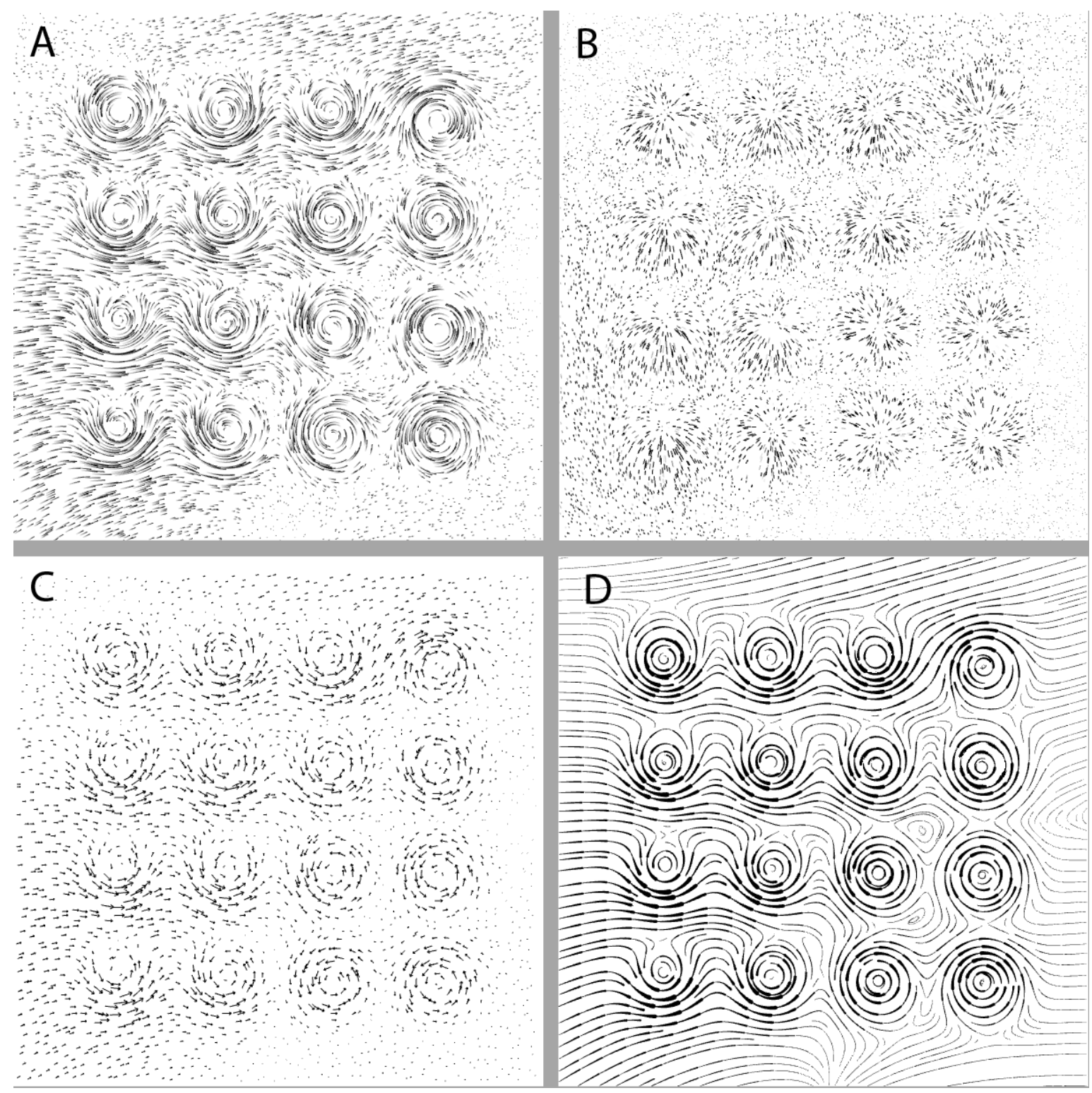

Figure 1: Four representations of the circular flow pattern. A) Animated streamlets. B) Animated orthogonal particles. C) A jittered grid of arrows. D) Equally spaced streamlines.

Ware and Plumlee [2013] evaluated randomly placed animated streamlines against static versions of the same streamlines and a glyph-based visualization. The task involved judging the perception of speed and direction (not pattern perception). They found the animated view better for the wind direction. They also obtained subjective assessments of the ability of animated flows to represent patterns using Likert scales. Animated streamlines were generally rated highly. However, the tested alternatives did not include equally spaced streamlines (probably the best static method), nor did they include an arrow grid.

\subsection{Vector field components}

In considering why motion might be more effective at revealing patterns in vector fields it is useful to decompose vectors into three parts: the magnitude (speed), the orientation, and the vector sign. A streamline can show orientation, but it will be ambiguous without an arrow head or other graphical device to show direction along the streamline, this is the vector sign. Animation can be used to show all of these components. The speed can be mapped to the speed of motion, the direction motion can show both the orientation and the vector sign. If streamlines are used to show orientation, color or line thickness can be used to show speed, and arrow heads, or animation can be used to show the vector sign. In the case of arrow grids, the length of the arrows is commonly used to represent the speed or vector magnitude. For static representations, various authors [Bertin, 1983; Ware, 2008] have suggested that heavy headed arrows or teardrop shapes may be better than conventional arrowhead for showing the vector sign.

\subsection{Perception of moving patterns}

We are aware of no prior empirical work, either in the vision science literature, or in the applied literature that directly compares 


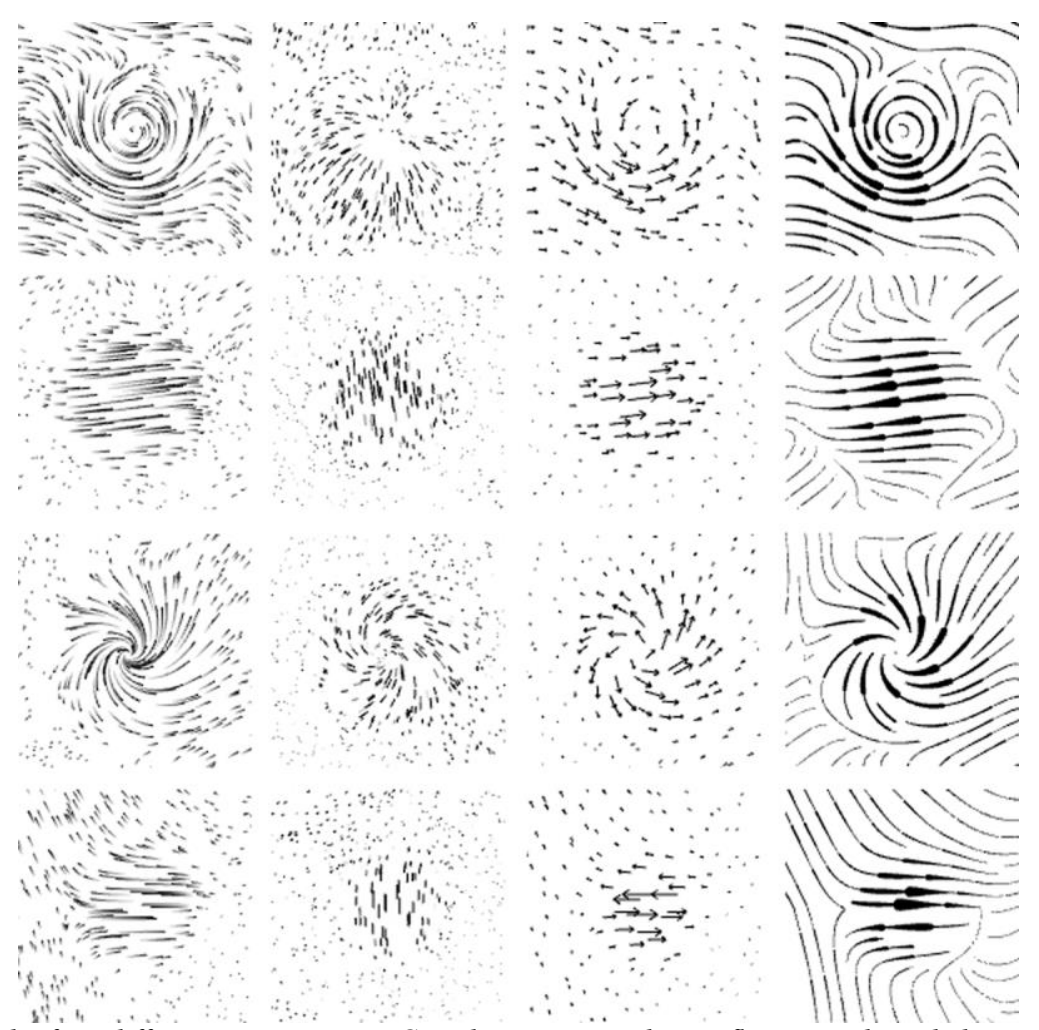

Figure 2: The rows show the four different test patterns: Circular rotations, linear flow, spirals and shear. The columns show the four rendering methods used: animated streamlets, animated orthogonal, arrow grid, equally spaced streamlines.

perception of static flow patterns to similar moving patterns. However, there are some lines of research that are relevant, albeit somewhat indirectly. There has been work in the area of visual search comparing motion to other cues, such as color or shape. Motion has been found to be preattentive allowing for increased accuracy and faster reaction times [Nothdurft, 1993; Triesman, 1985]. However, studies that have evaluated difference between motion and static stimuli have done so for specific features (i.e. color and line orientation). The stimuli themselves often differ greatly from one another. There is also evidence to suggest that motion is more effective at directing and capturing attention compared to non-moving stimuli [Bartram et al. 2003]. However, there are also search asymmetries; for example, moving targets are more readily found among static targets than the reverse case [Royden et al, 2001] and this suggests that if flow data are animated some patterns will be easier to perceive than others. Also relevant is work by McLeod et al [McLeod et al. 1988] who investigated visual search for target items defined by a conjunction of movement and form. They found that sometimes such a conjunction search could be fast. This and other studies have been taken as evidence operation of a movement filter in the visual system [Wolfe and Horowitz, 2004]. Gestalt theory of perceptual grouping states that objects of a common fate are group together [Wertheimer, 1938; McLeod et al. 1988]. Based on this theory it is expected that all stimuli moving in the same direction will be seen as one unit making the detection of a target moving opposite the group easier. This suggests that moving stimuli will activate the motion filter allowing for a more efficient visual search and faster recognition of anomalous targets moving in a different direction.

A number of researchers have compared visual search for different patterns of moving targets. Expanding patterns of dots are perceived more readily than contracting patterns [Franconeri and Simons, 2003]. Also, it is easier to see patterns against coherent motion, rather than random motion [Royden et al, 2001]. The size of patterns moving in groups can be estimated more accurately than if the same patterns are distributed randomly in space. It is possible, under certain conditions, to perceive animated flow in separate layers [Langer et al. 2005] but if the motion in the two layers is similar, the layers become perceptually fused [Masson et al, 1999].

Our study evaluates the effects of moving patterns compared to stimuli that are static representations of the same patterns. This allows for a direct comparison and an increased understanding of the advantages and disadvantages of incorporating motion into visualizations of vector field data. We conducted two experiments with different tasks, but the same set of representation methods, both static and animated. In the first, the participant's task was to detect a target pattern in a simple simulated flow field. This is similar to study by Pilar and Ware [2013] to evaluate alternative static representation methods. The second was based on Laidlaw et al's [2005] advection pathway tracing task where participants had to estimate the path of an imaginary particle deposited in a flow field. Our hypothesis was that animated flow patterns would speed up visual search for anomalous patterns and would improve advection pathway tracing.

\section{Experiment 1: Pattern Detection}

The approach we took was to generate simplified artificial vector fields in which various patterns were artificially constructed. The task of the study participant was to find an exceptional pattern in a 
set of similar patterns. In all cases the key differences lay in the vector sign. For example, a participant might be asked if there is a clockwise rotation in a set of patterns having counter-clockwise rotation.

\section{We compared four flow visualization methods (Figure 1).}

Animated streamlets (AS). Animated streamlets are short streamlines that advect through the flow field. We gave ours opaque heads and transparent tails. Animation speed and the streamlet length showed velocity. To maintain continuity, individual traces fade after a certain interval and are replaced by a new trace at a randomly determined location. The effect is similar to oriented line integral convolution (Wegenkittl, 1997).

Animated orthogonal particles (AO). Particles in motion that are elongated orthogonally to the direction of movement through a vector field direction may provide stronger motion cues than traces that are aligned tangentially to the flow [Bachthaler and Weiskopf, 2008] We therefore included this as a condition, representing advected particles using short line segments orthogonal to the flow direction. Animation speed and line length showed velocity.

Static Arrow Grid (AG). Although there is now substantial evidence suggesting that gridded arrows are not a good way of representing vector fields, this is still the most common method used in practice as any scan of the scientific and technical literature will show. Arrow length represented velocity.

Static Equally spaced streamlines (JL). Studies suggest that this may be the most effective method for visualization 2D flow patterns [Laidlaw et al, 2005; Pineo and Ware, 2008]. We used the Jobard and Lefer [1997] algorithm to generate the streamlines and created tear drop patterns along the streamlines to show the directionality. The teardrop shapes were scaled according to velocity.

\subsection{Test patterns}

There were four test patterns always in a $4 \times 4$ grid. Examples are given in Figure 2. In all cases a smooth random background vector field was first constructed by summing five Gaussian blended randomly oriented and positioned vector patterns. Superimposed on this was one of four test patterns. Each of these was generated by multiplying a simple pattern by a Gaussian and adding the result to the background flow. Note that all speeds are arbitrary, because the patterns were artificial. The parameters defining the following patterns are given in units of screen pixels. Targets appeared on $50 \%$ or the trials.

Circular rotations. The patterns showed counter clockwise rotation, except that on half the trials one of the patterns (the target) was a clockwise rotation. The position of this was randomly determined. The patterns had a tangential speed given by $r e^{-r^{2} / 576}$

Linear flow. The patterns showed leftward motion, except on half the trials, one of the patterns (the target) was rightward. A direction was selected randomly between $+/-45$ deg of horizontal. Speed was calculated using $r e^{-r^{2} / 576}$

Spirals. The patterns were inward spirals, except on half the trials one of the patterns (the target) was an outward spiral. Motion was computed as the sum of circular and radial components. The circular component was given by $r e^{-r^{2} / 384}$ and the radial component was computed using the same expression $r e^{-r^{2} / 384}$

Shear. The upper half of each pattern showed leftward motion and the lower half of each pattern showed rightward motion, except on half the trials one of the patterns (the target) had the reverse pattern. Horizontal speed was given by $r e^{-r^{2} / 288}$. Above the midpoint flow was the left and below the midpoint flow was to the right, unless it was a target pattern in which case this was reversed. In addition, the vertical flow of the background was reduced by being multiplied by $1-r e^{-r^{2} / 576}$

The test pattern was rendered in a $600 \times 600$ pixel window. This measured $16.25 \mathrm{~cm}$ in width and height. An attempt was made to seat subjects such that the viewing distance was $57 \mathrm{~cm}$ (meaning that one $\mathrm{cm}$ subtended approximately one degree of visual angle).

\subsection{Rendering details}

Because we were specifically interested in whether motion was responsible for any results we were careful to remove aspects of the rendering methods that might make the task easier independent of motion. For example, animated particles tend to cluster in sink regions of a flow and be sparse in source regions. So simple visual density could be used to distinguish sources from sinks and motion might be irrelevant. To remove this effect we first computed random starting points for streamlets, then we backed them up using reverse advection for a number of steps equal to half the life of a streamlet. These backed up points were used for the actual starting points.

Streamlets: 6000 streamlets were rendered as animated polylines opaque at the head and transparent at the tail (Ware and Plumlee, 2013). In the fastest parts of the patterns the streamlets advanced by 30 pixels/s. The length of the streamlets provided an additional cue to flow speed (maximum length was 60 pixels). To avoid popping effects, streamlets were faded in when they were born and faded out at the end of their lives.

Orthogonal animated particles: 1000 animated particles were rendered. In the fastest parts of the patterns the particles advanced by $30 \mathrm{pixels} / \mathrm{s}$. The maximum line length (orthogonal to the flow) was 8 pixels. The length of the particles provided an additional cue for flow speed to make this comparable to streamlets. However, lengths were necessarily shorter.

Arrow grid: A 50 x50 jittered grid of arrows was drawn (12 pixel spacing on average). The arrow length was proportional to the speed with a maximum length of 20.0 pixels. The width of the arrow head was one third of the length. The shaft of the arrow was centered on the jittered grid point.

Equally spaced streamlines: The Jobard and Lefer (1997) algorithm was used to compute streamlined. Line spacing varied between 8 and 16 pixels. Teardrop shapes along the streamlines were used to show speed and direction. These were scaled with speed.

\subsection{Task}

On each trial the participant's task was to determine if there was an anomalous pattern (as described in the test patterns section). They responded using one of two specially marked keys on the keyboard. [Yes] (overlaid on the V-key) if the pattern was present and [No] (overlaid on the N-key) if it was not. Participants normally kept their left index finger on the [No] key and their right index finger 
on the [Yes] key. If they made an error responding they heard an auditory beep through headphones.

\subsection{Participants}

There were 14 participants (12 female and 2 male). They were all paid for participating.

\subsection{Procedure}

There were 16 conditions ( 4 representations $\times 4$ test patterns). Trials were given in blocks of 8 for each condition. On half of the trials within a given block (randomly determined) the target was present and on half the target was absent. The entire set of conditions was given in a different random order for each participant and there was one replication of the entire set, yielding 16 trials per condition per participant. Prior to the experiment proper, a training session was given where participants ran through the conditions in a random order with two trials per condition. In this phase participants were given directions on what to look for and corrected when they made an error.

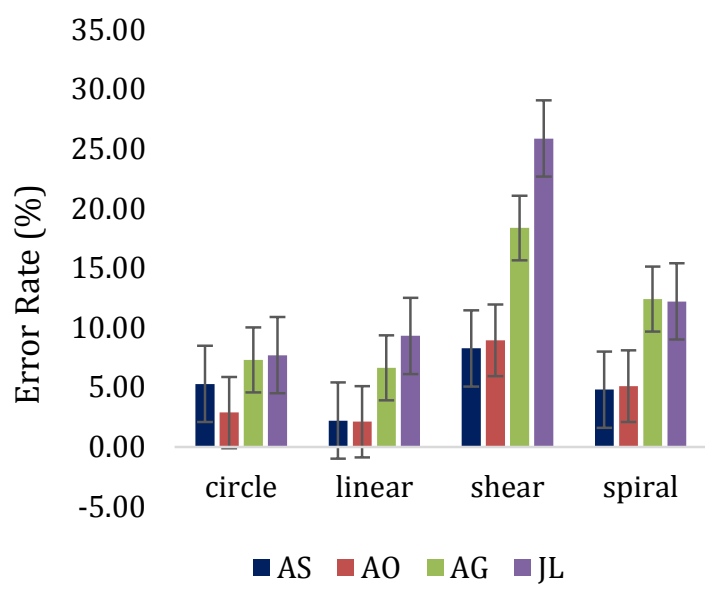

Figure 3. Mean error rates for Experiment 1. AS: Animated streamlets. AO: Animated orthogonal particles. AG: Arrow Grid. JL: Equally spaced streamlines using the Jobard and Lefer algorithm.

\subsection{Results from Experiment 1}

The main results are summarized in Figures 3 and 4. To evaluate possible differences between moving and static stimuli a repeatedmeasures ANOVA with factors of pattern (circle, linear flow, shear, and spiral), representation (animated streamlets, animated orthogonal particles, arrows, and equally spaced streamlines) and target (absent or present) was conducted using the average number of errors made and average response time as dependent variables. For number of errors there were significant main effects of representation (Greenhouse-Geisser corrected $\mathrm{F}[1.589,19.07]=$ $\left.14.302, \mathrm{p}<.0001, \eta^{2}=.544\right)$, pattern $(\mathrm{F}[3,36]=17.223, \mathrm{p}<.0001$, $\left.\eta^{2}=.589\right)$, and target $\left(F[1,12]=17.906, p<.001, \eta^{2}=.599\right)$.

Pairwise comparisons using the Bonferroni correction for multiple comparisons showed that the two animated representations yielded significantly greater accuracy than both static representations (Figure 3). The animated representation did not differ significantly from one another, neither did the static representations. Specifically: Animated streamlets was significantly better than arrow $(\mathrm{p}<.003)$ and equally spaced streamlines $(\mathrm{p}<.002)$, animated orthogonal particles was better than arrow $(\mathrm{p}<.001)$, and equally spaced streamlines $(\mathrm{p}<.006)$.

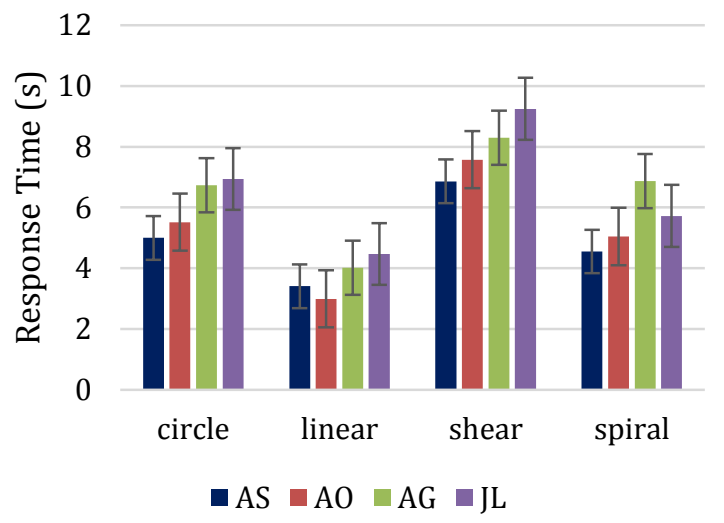

Figure 4. Mean response times for Experiment 1. AS: Animated streamlets. AO: Animated orthogonal particles. AG: Arrow Grid. JL: Equally spaced streamlines using the Jobard and Lefer algorithm.

Pairwise comparisons of pattern using Bonferroni correction found the percent of errors made for the pattern shear was significantly greater than circle $(p<.001)$, linear flow $(p<.002)$, and spiral $(p<$ .027). Pairwise comparisons of target found that the numbers of errors made for the target absent condition $(M=0.046)$ was significantly different from the target present condition $(\mathrm{p}=0.001$, $\mathrm{M}=0.129$ ). In addition, a two-way interaction of pattern by representation was found (Greenhouse-Geisser corrected $\mathrm{F}[3.519$, $\left.42.232]=2.795, \mathrm{p}<.044, \eta^{2}=.189\right)$. An analysis of simple main effects found that although the representation of shear produced the highest number of errors this is dependent on the combined representation. The combination of shear and animated streamlets $(\mathrm{M}=0.083)$ significantly different from the combination of shear and arrows $(\mathrm{p}=0.016, \mathrm{M}=0.184)$ and the combination of shear and equally spaced streamlines $(\mathrm{p}=0.012, \mathrm{M}=0.259)$ but not different from the combination of shear and animated orthogonal particles $(\mathrm{M}=0.089)$. This further supports our hypothesis that the animated representations produce fewer errors than the static representations. The pattern of shear only increased errors for the static representations but had no significant effect on the animated representations.

Evaluation of response time showed significant main effects of pattern (Greenhouse-Geisser corrected $\mathrm{F}[1.607,19.286]=37.751$, $\left.\mathrm{p}<.0001, \eta^{2}=.759\right)$, representation $(F[3,36]=11.237, \mathrm{p}<.0001$, $\left.\eta^{2}=.484\right)$, and target $\left(F[1,12]=63.498, p<.0001, \eta^{2}=.841\right)$.

Pairwise comparisons found that the two animated representations yielded significantly faster responses than both static representations (Figure 4). The animated representation did not differ significantly from one another, neither did the static representations. Specifically: animated streamlets was significantly faster than arrow $(p<.001)$ and equally spaced streamlines $(p<$ .015). Animated orthogonal particles was significantly different from arrow $(\mathrm{p}<.044)$ and equally spaced streamlines $(\mathrm{p}<.047)$. 
Pairwise comparisons of pattern found the response time for linear flow was significantly faster than circle $(p<.0001)$, shear $(p=$ $.0001)$, and spiral $(\mathrm{p}<.0001)$. Shear responses were significantly slower than circle $(\mathrm{p}<.008)$, and spiral $(\mathrm{p}<.001)$ responses. As seen in figure 6 the pattern of linear flow displayed the fastest response time whereas shear displays the slowest response time. The same is true for the static representations.

Overall, response times were significantly longer for the target absent condition than the target present condition. Pairwise comparisons of target using Bonferroni correction found that the response times for the target absent condition $(\mathrm{M}=6.736)$ was significantly longer than the target present condition $(p=0.0001$, $\mathrm{M}=4.920)$. This is in agreement with theories of visual search since a search can be terminated when a target is found, but if the target is absent all the potential targets must be examined.

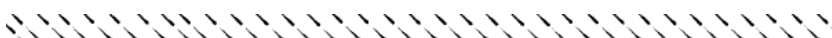
N.

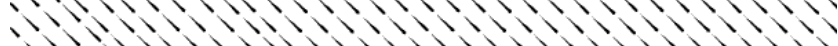

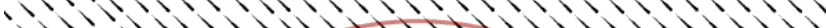

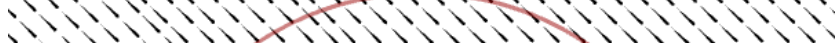

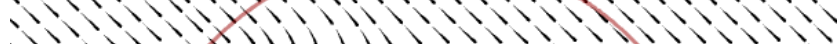
八

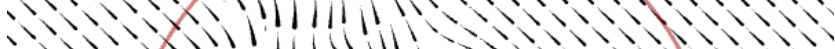
NI II

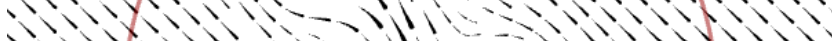
N1 № NIIIII $\therefore N=$ = $\therefore N$ $\therefore N$ NIIININIVE- = NIIINININ

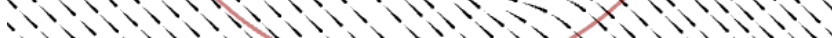

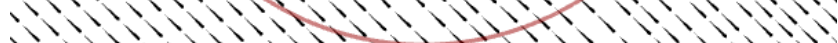

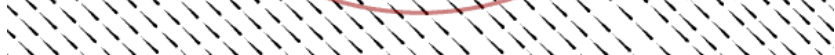

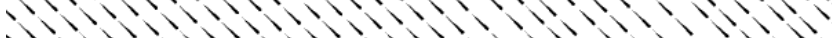

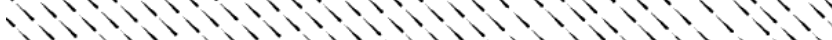
Figure 5. A screen from Experiment 2. The participant has placed the dot on the circumference of the circle to indicate where a particle dropped at the center would exit the circle.

\section{Experiment 2: Advection Pathway Tracing}

The second experiment used advection pathway tracing as a task. This was developed by Laidlaw et al [Laidlaw et al, 2005] for the experimental comparison of static vector fields. In this method, a dot is placed in the center of the vector field, with a circle surrounding it. The participant's task is to estimate where a particle dropped at the center would exit the circle. They indicate this with a mouse click.

\subsection{Display patterns}

An example of a test screen is given in Figure 5. The vector field was constructed by first selecting a random direction and filling all cells in the grid with that value. Added to this were 20 Gaussian blended randomly oriented and positioned patterns. Note that all speeds are arbitrary, because the patterns were artificial. The final pattern was tested using a second order Runge-Kutta method to determine that an advection pathway existed from the center to the circle. If none existed a new pattern was generated until a viable one was found. The test pattern was rendered in a 600x600 pixel window. This measured $16.25 \mathrm{~cm}$ in width and height. The radius of the circle was $5.8 \mathrm{~cm}$. In the animated condition, the average speed was such that a particle crossing the center point took 3.5 seconds on average to cross the circle boundary (about $1.6 \mathrm{deg} / \mathrm{s}$ ).

\subsection{Participants}

There were 15 participants, 12 female, and 3 male all of whom were paid for participating in the experiment.

\subsection{Conditions and trials}

There were four conditions consisting of the same set of representations as the first experiment. Trials were given in blocks of 15 for each condition. The entire set of conditions was given in a different random order for each participant. Prior to the experiment proper, a training session was given where participants ran through the conditions in a random order with two trials per condition. In this phase participants were given directions how to use the interface.

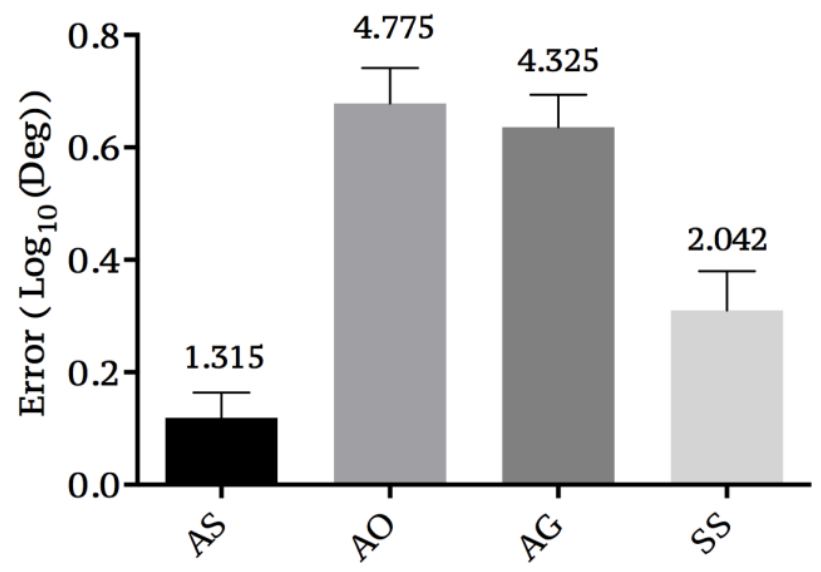

Figure 6: Mean log errors for Experiment 2 Anti-log values are provided above each bar. AO: Animated orthogonal particles. AG: Arrow Grid. JL: Equally spaced streamlines using the Jobard and Lefer algorithm

\subsection{Results from Experiment 2}

To evaluate the possible differences between moving and static stimuli a repeated-measures ANOVA with the factors of representation (animated streamlets, animated orthogonal particles, arrows, and equally spaced streamlines) and number of repetitions (15 repetitions of each representation) was conducted using the $\log 10$ of the degrees of error and response time in seconds as dependent variables. The log transform was used to create more uniform variance.

The results are summarized in Figures 6 and 7. For error a significant main effects of representation $(F[3,42]=33.862, p<$ $\left..0001, \eta^{2}=.707\right)$ was found. Pairwise comparisons of representation using Bonferroni correction found the error for animated streamlets was significantly lower than for animated orthogonal particles $(p<.0001)$, arrows $(p<.0001)$ and equally spaced streamlines $(p=.006)$. Equally space streamlines produced fewer errors than animated orthogonal particles $(\mathrm{p}<.0001)$ and the arrow grid $(p<0.0001)$. In other words, the representation of animated streamlets had the smallest error compared to the other three representations. Performance for the animated orthogonal particles was poorest but not significantly different from arrows. 
These results suggest that animated streamlets are the best representation for this task; with equally spaced streamlines being the next best option.

For response time a significant main effects of representation $(\mathrm{F}[3$, 42] $=40.340, p<.0001, \eta^{2}=.742$ ) was found. Pairwise comparisons of representation using Bonferroni correction found the response times for animated streamlets was significantly faster than for animated orthogonal particles $(p<.0001)$ but slower than equally spaced streamlines $(\mathrm{p}=.006)$ but not significantly different from arrows. Responses with Animated orthogonal particles were significantly slower than with equally spaced streamlines $(\mathrm{p}<$ $.0001)$ and the arrow grid $(\mathrm{p}<.0001)$. Arrows were also slower than equally spaced streamlines $(p=.015)$.

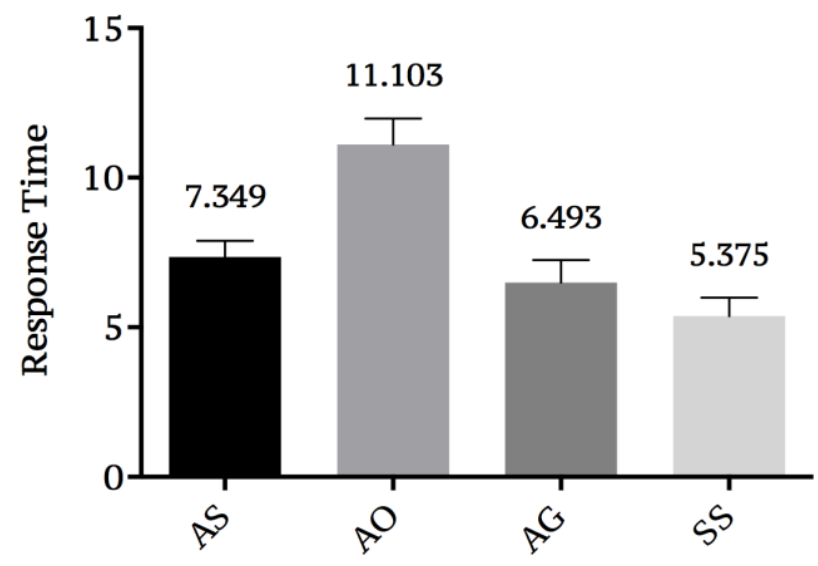

Figure 7: Mean response times for Experiment 2.

\section{Discussion}

In the pattern search task (Experiment 1) errors for both of the animated representations were approximately half those for the static representations. This provides strong support for our hypothesis that animation is the most effective way of showing flow direction. Responses were also faster for the animated views. There were no significant differences between the two animated methods and no significant differences between the two static methods.

For the advection task the animated streamlet method was the clear winner overall. It resulted in average errors approximately one third as large as for the arrow grid. The next best method was the static equally spaced streamlines representation. The arrows and the animated orthogonal particles produced equally poor performance. Our finding that equally spaced streamlines produces lower errors than an arrow grid is in agreement with Laidlaw et al's [2005] prior finding and theory [Ware, 2008; Pineo and Ware, 2008]. We have not, of course, tested all possible animated representations against all static ones, but the size of the effects suggests that the advantage of animated representations is robust, especially for animated streamlets.

As noted in our introduction, there are asymmetries in visual search for moving patterns; for example expanding (looming) patterns are seen more readily than contracting (receding) patterns [Franconeri and Simons, 2003]. In the first experiment one of the patterns, a clockwise expanding spiral was compared to a counter-clockwise expanding spiral and results may have been different for contraction spirals. The other three patterns, however, were neither expanding nor contracting, and the results in all cases showed the moving patterns generate fewer errors than the static patterns. As a more general point certain kinds of patterns in animated flow will undoubtedly stand out more clearly than others. But this is certainly also the case for static flow visualizations, as the results show, and currently there is no evidence that animation is worse in this respect than static views.

There are, of course, disadvantages to using animated vector fields. The principal of these is the computational cost of generating moving patterns. In addition the method is not suitable for print media and this may lead to the use of different representations for print and for screen. A third disadvantage may relate to the representation of speed of flow. Some vector fields have a large dynamic range. For example, in the case of winds we may care about both about the $200 \mathrm{kph}$ winds of a hurricane and the pattern of light $5 \mathrm{kph}$ winds in other parts of the globe. If animation speed is made proportional to wind speed, some particles may be moving too fast or two slow for the movement patterns to be readily perceived. This problem is also common to other representation methods, such as using arrow length to show speed. One solution is to use a non-linear transformation of speed, such as a square root function [Ware and Plumlee, 2013] This means that it will be difficult to judge relative speed from the speed of streamlet motion, but we believe that judgements of motion speed are generally poor and that methods such as color coding, or custom glyphs [Pilar and Ware, 2013] will be the best way of representing flow speed.

It was remarked by one of the study participants that they could perform the advection pathway task in the animated condition by picking a streamlet passing through the center dot of the target region and visually tracking it to the edge of the circle. This can perhaps explain the superior accuracy in this condition. But this method could also have been applied with the orthogonal particles but errors were much greater in that condition, so clearly other factors were involved.

There are other advantages of animated streamlets that have previously been remarked, in particular that animation employs a separate visual "channel" meaning that when animation is used there is less visual interference between an animated overlay and other background information. In addition, research shows that under certain circumstances multiple animated layers can be perceived, albeit with limitations [Langer et al., 2006]. Because our ability to perceive relative speed is a function of average speed [Masson et al 2005] having only a single moving layer is likely to result clear visual separation between a background static layer and the animated layer, although slower speeds may be less distinct than faster speeds. This can be useful in complex multi-layered displays where wind speeds and direction are overlaid on other information such as atmospheric pressure or temperature [Ware and Plumlee, 2013]. For dynamic vector fields, animated streamlets, or pathlets are becoming quite common, our results suggest that they can also be a good choice for steady vector fields.

\section{Acknowledgements}

We gratefully acknowledge funding from the US Department of Energy DE-SC0012438.

\section{References}

BachthaleR, S., \& Weiskopf, D. 2008. Animation of orthogonal 
texture patterns for vector field visualization. Visualization and Computer Graphics, IEEE Transactions on, 14(4), 741-755.

Bartram, L., Ware, C. AND CAlvert, T. 2003. Moticons: detection, distraction and task. International Journal of HumanComputer Studies, 58(5), pp.515-545.

Berger, S., \& GRÖLler, E. 2000. Color-Table Animation of Fast Oriented Line Integral Convolution for Vector Field Visualization. In WSCG.

Bertin, J., 1983. Semiology of graphics: diagrams, networks, maps.

Cabral, B., \& Leedom, L. C. (1993, September). Imaging vector fields using line integral convolution. In Proceedings of the 20th annual conference on Computer graphics and interactive techniques (pp. 263-270). ACM.

Franconeri, S.L. AND Simons, D.J., 2003. Moving and looming stimuli capture attention. Perception \& psychophysics, 65(7), pp.999-1010.

Haroz, S. AND Whitney, D., 2012. How capacity limits of attention influence information visualization effectiveness. Visualization and Computer Graphics, IEEE Transactions on, 18(12), pp.2402-2410.

Huber, D.E. AND Healey, C.G., 2005, October. Visualizing data with motion. In Visualization, 2005. VIS 05. IEEE (pp. 527-534). IEEE.

JobARD, B., \& LEFER, W. 1997. The motion map: efficient computation of steady flow animations. In Visualization'97., Proceedings (pp. 323-328). IEEE.

Laidlaw, D. H., Kirby, R. M., Jackson, C. D., DavidSon, J. S., Miller, T. S., DA Silva, M., ... \& TARR, M. J. (2005). Comparing $2 \mathrm{D}$ vector field visualization methods: A user study. Visualization and Computer Graphics, IEEE Transactions on, 11(1), 59-70.

LAnger, M. S., Pereira, J., \& Rekhi, D. (2006). Perceptual limits on 2D motion-field visualization. ACM Transactions on Applied Perception (TAP), 3(3), 179-193.

Laramee R. S., Hauser H., Doleisch H., Vrolijk B., Post F. H., WEISKOPF D.: The state of the art in flow visualization: Dense and texture-based techniques. Computer Graphics Forum 23, 2 (2004), 203-221. 2

LARAMEE, R. S. 2002, Interactive 3d flow visualization using a streamrunner. In Conference on Human Factors in Computing Systems: CHI'02 extended abstracts on Human factors in computing systems (Vol. 20, No. 25, pp. 804-805).

Lefer W., Jobard, B. AND Leduc, C. 2004 High-Quality Animation of 2D Steady Vector Felds," IEEE Trans. Visualization and Computer Graphics 10(1), pp. 2-14.

LiU, Z., Cai, S., Swan, J.E., MoOrhead, R.J., Martin, J.P. AND JANKUN-KELLY, T.J., 2012. A 2D flow visualization user study using explicit flow synthesis and implicit task design. Visualization and Computer Graphics, IEEE Transactions on, 18(5), pp.783-796.

Livingstone, M.S. And Hubel, D.H., 1987. Psychophysical evidence for separate channels for the perception of form, color, movement, and depth. The Journal of Neuroscience, 7(11), pp.3416-3468.

Masson, G., Mestre, D. And Stone, L. 1999. Speed tuning of motion segmentation and discrimination. Vision Research 39, 256, 4297-4308.

MAX, N., \& BECKER, B. (1996). Flow visualization using moving textures. In NASA Conference Publication (pp. 77-88). NASA..

MCLeOD, P., Driver, J. AND CRISP, J., 1988. Visual search for a conjunction of movement and form is parallel. Nature.

Newsome, W. T., AND PARE. E. B. 1988 A selective impairment of motion perception following lesions of the middle temporal visual area (MT). The Journal of Neuroscience 8, no. 6 2201-2211.

NothDuRFT, H.C., 1993. The role of features in preattentive vision: Comparison of orientation, motion and color cues. Vision research, 33(14), pp.1937-1958.

Pilar, D. H., \& WARE, C. (2013). Representing flow patterns by using streamlines with glyphs. IEEE Transactions on Visualization \& Computer Graphics, (8), 1331-1341.

PINEO, D., \& WARE, C. 2008. Neural modeling of flow rendering effectiveness. In Proceedings of the 5th symposium on Applied perception in graphics and visualization (pp. 171-178). ACM.

Royden, C.S., Wolfe, J.M. AND KLemPen, N., 2001. Visual search asymmetries in motion and optic flow fields. Perception \& Psychophysics, 63(3), pp.436-444.

Sobel, J. S., Forsberg, A., Laidlaw, D. H., Zeleznik, R. C., Keefe, D. F., Pivkin, I., Karniadakis, G.E. Richardson, P. \& SwartZ, S. 2004. Particle flurries. Computer Graphics and Applications, IEEE, 24(2), 76-85.

Telea, A., \& VAN WiJK, J. J. (1999, October). Simplified representation of vector fields. In Proceedings of the conference on Visualization'99: IEEE Computer Society Press. pp. 35-42).

Treisman, A., 1985. Preattentive processing in vision. Computer vision, graphics, and image processing, 31(2), pp.156-177.

VAN WIJK, J.J., 1993. Flow visualization with surface particles. Computer Graphics and Applications, IEEE, 13(4), pp.18-24.

WARE, C. 2008. Toward a perceptual theory of flow visualization. Computer Graphics and Applications, IEEE, 28(2), 6-11.

Ware, C., Kelley, J. G., \& Pilar, D. (2014). Improving the Display of Wind Patterns and Ocean Currents. Bulletin of the American Meteorological Society, 95(10), 1573-1581.

Ware, C., \& Plumlee, M. D. (2013). Designing a better weather display. Information Visualization, 12(3-4), 221-239.

WERTHEIMER, M., 1938. Laws of organization in perceptual forms.

Wolfe, J.M. AND Horowitz, T.S., 2004. What attributes guide the deployment of visual attention and how do they do it? Nature reviews neuroscience, 5(6), pp.495-501 